Editorials

\title{
A New Sequence Space Defined by Spectrum Operator and Musielak-Orlicz Function
}

\author{
${ }^{1}$ Ayhan Esi and ${ }^{2}$ Nagarajan Subramanian \\ ${ }^{I}$ Department of Mathematics, Adiyaman University, 02040, Adiyaman, Turkey \\ ${ }^{2}$ Department of Mathematics, SASTRA University, Thanjavur-613 401, India
}

Article history

Received: 17-04-2017

Revised: $18-05-2017$

Accepted: 25-05-2017

Corresponding Author:

Ayhan Esi

Department of Mathematics,

Adiyaman University, 02040,

Adiyaman, Turkey

Email: aesi23@hotmail.com

\section{Introduction}

Throughout $w, \chi$ and $\Lambda$ denote the classes of all, gai and analytic scalar valued single sequences, respectively. We write $w^{3}$ for the set of all complex triple sequences $\left(x_{m n k}\right)$, where $m, n, k \in \mathbb{N}$, the set of positive integers. Then, $w^{3}$ is a linear space under the coordinate wise addition and scalar multiplication.

We can represent triple sequences by matrix. In case of double sequences we write in the form of a square. In the case of a triple sequence it will be in the form of a box in three dimensional case.

Some initial work on double series is found in Apostol (1978) and double sequence spaces is found in Hardy (1917; Deepmala and Mishra, 2016; Deepmala et al., 2016) and many others. Later on investigated by some initial work on triple sequence spaces is found in Sahiner et al. (2007; Esi, 2014; Esi and Necdet Catalbas, 2014; Esi and Savas, 2015; Subramanian and Esi, 2015; Prakash et al., 2016) and many others.

Let $\left(x_{m n k}\right)$ be a triple sequence of real or complex numbers. Then the series $\sum_{m, n, k=1}^{\infty} x_{m n k}$ is called a triple series. The triple series $\sum_{m, n, k=1}^{\infty} x_{m n k}$ give one space is said to be convergent if and only if the triple sequence $\left(S_{m n k}\right)$ is convergent, where:

$$
S_{m n k}=\sum_{i, j, q=1}^{m, n, k} x_{i, j q}(m, n, k=1,2,3, \ldots)
$$

A sequence $x=\left(x_{m n k}\right)$ is said to be triple analytic if:

$$
\sup _{m, n, k}\left|x_{m n k}\right|^{\frac{1}{m+n+k}}<\infty
$$

The vector space of all triple analytic sequences are usually denoted by $\Lambda^{3}$. A sequence $x=\left(x_{m n k}\right)$ is called triple entire sequence if:

$$
\left|x_{m n k}\right|^{\frac{1}{m+n+k}} \rightarrow 0 \text { as } m, n, k \rightarrow \infty
$$

The vector space of all triple entire sequences are usually denoted by $\Gamma^{3}$ : The spaces $\Lambda^{3}$ and $\Gamma^{3}$ are metric spaces with the metric:

$d(x, y)=\sup _{m, n, k}\left\{\left|x_{m n k}-y_{m n k}\right|^{\frac{1}{m+n+k}}: m, n, k: 1,2,3, \ldots\right\}$

For all $x=\left\{x_{m n k}\right\}$ and $y=\left\{y_{m n k}\right\}$ in $\Gamma^{3}$ : Let $\phi=\{$ finite sequences\}.

Consider a triple sequence $\mathrm{x}=\left(x_{m n k}\right)$, The $(m, n, k)^{\text {th }}$ section $x[m, n, k]$ of the sequence is defined by $x[m, n, k]$ $=\sum_{i, j, q=0}^{m, n, k} x_{i j q} \mathfrak{I}_{i j q}$ for all $m, n, k \in \mathbb{N}$ :

$$
\mathfrak{J}_{i j q}=\left[\begin{array}{ccccc}
0 & 0 & \ldots 0 & 0 & \ldots \\
0 & 0 & \ldots 0 & 0 & \ldots \\
. & & & & \\
\cdot & & & & \\
. & & & & \\
0 & 0 & \ldots 1 & 0 & \ldots \\
0 & 0 & \ldots 0 & 0 & \ldots \\
. & . & \ldots & . & \ldots \\
. & . & \ldots . & . & \ldots .
\end{array}\right]
$$

with 1 in the $(i, j, q)^{\text {th }}$ position and zero otherwise. 
A sequence $x=\left(x_{m n k}\right)$ is called triple gai sequence if $\left((m+n+k) !\left|x_{m n k}\right|\right)^{\frac{1}{m+n+k}} \rightarrow 0$ as $m, n, k \rightarrow 1$. The triple gai sequences will be denoted by $\chi^{3}$. The notion of difference sequence spaces (for single sequences) was introduced by Kizmaz (1981) as follows:

$$
Z(\Delta)=\left\{x=\left(x_{k}\right) \in w:\left(\Delta x_{k}\right) \in Z\right\}
$$

for $Z=c, c_{0}$ and $\ell_{\infty}$, where $\Delta x_{k}=x_{k}-x_{k+1}$ for all $k \in \mathbb{N}$.

Here $c, c_{0}$ and $\ell_{\infty}$ denote the classes of convergent, null and bounded scalar valued single sequences respectively. The difference sequence space $b v_{p}$ of the classical space $\ell_{p}$ is introduced and studied in the case 1 $\leq p \leq \infty 1$ by Basar and Altay and in the case $0<p<1$ by Altay and Basar. The spaces $c(\Delta), c_{0}(\Delta), \ell_{\infty}(\Delta)$ and $b v_{p}$ are Banach spaces normed by:

$$
\begin{aligned}
& \|x\|=\left|x_{1}\right|+\sup _{k \geq 1}\left|\Delta x_{k}\right| \text { and }\|x\|_{b v_{p}} \\
& =\left(\sum_{k=1}^{\infty} \|\left. x_{k}\right|^{p}\right)^{1 / p},(1 \leq p \leq \infty)
\end{aligned}
$$

Later on the notion was further investigated by many others. We now introduce the following difference double sequence spaces defined by:

$$
Z(\Delta)=\left\{x=\left(x_{m n}\right) \in w^{2}:\left(\Delta x_{m n}\right) \in Z\right\}
$$

where, $Z=\Lambda^{2}, \chi^{2}$ and $\Delta x_{m n}=\left(x_{m n}-x_{m n+1}\right)-\left(x_{m+1 n}-x_{m+1 n+1}\right)$ $=x_{m n}-x_{m n+1}-x_{m+1 n}+x_{m+1 n+1}$ for all $m, n \in \mathbb{N}$. The generalized difference double notion has the following representation: $\Delta^{m} x_{m n}=\Delta^{m-1} x_{m n}-\Delta^{m-1} x_{m n+1}-\Delta^{m-1} x_{m+1 n}+$ $\Delta^{m-1} x_{m+1 n+1}$ and also this generalized difference double notion has the following binomial representation: $\Delta^{m} x_{m n}$ $=\sum_{i=0}^{m} \sum_{j=0}^{m}(-1)^{i+j}\left(\begin{array}{l}m \\ i\end{array}\right)\left(\begin{array}{l}m \\ j\end{array}\right) x_{m+i, n+j}$

Let $w^{3}, \chi^{3}\left(\Delta_{m n k}\right)$ and $\Lambda^{3}\left(\Delta_{m n k}\right)$ be denote the spaces of all, triple gai difference sequence space and triple analytic difference sequence space respectively and is defined as:

$$
\begin{aligned}
& \Delta_{m n k}=x_{m n k}-x_{m, n+1, k}-x_{m, n, k+1}+x_{m, n+1, k+1}-x_{m+1, n, k} \\
& +x_{m+1, n+1, k}+x_{m+1, n, k+1}-x_{m+1, n+1, k+1} \text { and } \Delta^{0} x_{m n k}=\left\langle x_{m n k}\right\rangle
\end{aligned}
$$

\section{Definitions and Preliminaries}

\section{Definition (Kamthan and Gupta, 1981)}

An Orlicz function is a function $M:[0, \infty) \rightarrow[0, \rightarrow)$ which is continuous, non-decreasing and convex with $M(0)=0, M(x)>0$, for $x>0$ and $M(x) \rightarrow \infty$ as $\mathrm{x} \rightarrow \infty$. If convexity of Orlicz function $\mathrm{M}$ is replaced by $M(x+$ $y) \leq M(x)+M(y)$; then this function is called modulus function.

Lindenstrauss and Tzafriri (1971) used the idea of Orlicz function to construct Orlicz sequence space:

$$
\ell_{M}=\left\{x \in w: \sum_{k=1}^{\infty} M\left(\frac{\left|x_{k}\right|}{\rho}\right)<\infty, \text { for some } \rho>0\right\}
$$

The space $\ell_{M}$ with the norm:

$$
\|x\|=\inf \left\{\rho>0: \sum_{k=1}^{\infty} M\left(\frac{\left|x_{k}\right|}{\rho}\right) \leq 1\right\}
$$

becomes a Banach space which is called an Orlicz sequence space. For $M(t)=t^{p}(1 \leq p<\infty 1)$, the spaces $\ell_{M}$ coincide with the classical sequence space $\ell_{p}$,

A sequence $f=\left(f_{m n k}\right)$ of Orlicz function is called a Musielak-Orlicz function (Musielak, 1983). A sequence $g=\left(g_{m n}\right)$ defined by:

$$
g_{m n}(v)=\sup \left\{|v| u-\left(f_{m n k}\right)(u): u \geq 0\right\}, m, n, k=1,2, \ldots
$$

is called the complementary function of a MusielakOrlicz function $f$. For a given Musielak-Orlicz function $f$, the Musielak-Orlicz sequence space $t_{f}$ is defined as follows:

$$
t_{f}=\left\{x \in w^{3}: I_{f}\left(\left|x_{m n k}\right|\right)^{1 / m+n+k} \rightarrow \text { 0as } m, n, k \rightarrow \infty\right\}
$$

where, $I_{f}$ is a convex modular defined by:

$$
I_{f}(x)=\sum_{m=1}^{\bar{\infty}} \sum_{n=1}^{\infty} \sum_{k=1}^{\infty} f_{m n k}\left(\left|x_{m n k}\right|\right)^{1 / m+n+k}, x=\left(x_{m n k}\right) \in t_{f}
$$

We consider $t_{f}$ equipped with the Luxemburg metric:

$$
d(x, y)=\sum_{m=1}^{\infty} \sum_{n=1}^{\infty} \sum_{k=1}^{\infty} f_{m n k}\left(\frac{\left|x_{m n k}\right|^{1 / m+n+k}}{m n k}\right)
$$

is an exteneded real number.

Let $X$ and $Y$ be Banach metric spaces and $T: X \rightarrow Y$ be a bounded linear operator. The set of all bounded linear operators on $X$ into itself is denoted by $B(X)$. The adjoint $T^{*}: X^{*} \rightarrow X^{*}$ of $T$ is defined by $\left(T^{*} \phi\right)(x)=\phi(T x)$ for all $\phi \in X^{*}$ and $x \in X$. Clearly, $T^{*}$ is a bounded linear operator on the dual space $X^{*}$.

Let $T: D(T) \rightarrow X$ a linear operator, defined on $D(T) \subset X$, where $D(T)$ denote the domain of $T$ and $X$ is a complex normed linear space. For $T \in B(X)$ we associate a complex number $\alpha$ with the operator $(T-\alpha I)$ denoted by $T_{\alpha}$ defined on the same domain $D(T)$, where $I$ is the identity operator. The inverse $(T-\alpha I)^{-1}$, denoted by $T_{\alpha}^{-1}$ is 
known as the resolvent operator of $T$. Many properties of $T_{\alpha}$ and $T_{\alpha}^{-1}$ depend on á and spectral theory is concerned with those properties. We are interested in the set of all $\alpha$ in the complex plane such that $T_{\alpha}^{-1}$ exists. Boundedness of $T_{\alpha}^{-1}$ is another essential property. We also detemine $\alpha^{\prime} s$, for which the domain of $T_{\alpha}^{-1}$ is dense in $X$.

A regular value is a complex number $\alpha$ of $T$ such that:

- $\quad\left(N_{1}\right) T_{\alpha}^{-1}$ exists

- $\quad\left(N_{2}\right) T_{\alpha}^{-1}$ is bounded and

- $\left(N_{3}\right) T_{\alpha}^{-1}$ is defined on a set which is dense in $X$

The resolvent set of $T$ is the set of all such regular values $\alpha$ of $T$, denoted by $\rho(T)$. Its complement is given by $C \backslash \rho(T)$ in the complex plane $C$ is called the spectrum of $T$, denoted by $\sigma(T)$. Thus the spectrum $\sigma(T)$ consists of those values of $\alpha \in C$, for which $T_{\alpha}$ is not invertible.

We discuss about the point spectrum, continuous spectrum, residual spectrum, approximate point spectrum, defect spectrum and compression spectrum. There are many different ways to subdivide the spectrum of a bounded linear operator. Some of them are motivated by applications to physics, in particular in quantum mechanics.

\section{Definition}

The point (discrete) spectrum $\sigma_{p}(T, X)$ is the set of complex number $\alpha$ such that $T_{\alpha}^{-1}$ does not exist. Further $\alpha \in p(T, X)$ is called the eigen value of $T$.

\section{Definition}

The continuous spectrum $\sigma_{c}(T, X)$ is the set of complex number $\alpha$ such that $T_{\alpha}^{-1}$ exists and satisfies $\left(N_{3}\right)$ but not $\left(N_{2}\right)$ that is $T_{\alpha}^{-1}$ is unbounded.

\section{Definition}

The residual spectrum $\sigma_{r}(T, X)$ is the set of complex number $\alpha$ such that $T_{\alpha}^{-1}$ exists (and may be bounded or not) but not satisfy $\left(N_{3}\right)$, that is the domain of $T_{\alpha}^{-1}$ is not dense in $X$.

This is to note that in finite dimensional case, continuous spectrum coincides with the residual spectrum and equal to the empty set and the spectrum consists of only the point spectrum.

Given a bounded linear operator $T$ in a Banach metric space $X$, we call a sequence $\left(x_{m n k}\right) \in X$ as a sequence for $T$ if:

$d(x, 0)=1 \Rightarrow\left\|x_{m n k}-0\right\|=1=\left\|x_{m n k}\right\|$

and:
$d(T x, 0)=1 \Rightarrow\left\|T x_{m n k}-0\right\|=\left\|T x_{m n k}\right\| \rightarrow 0$ as $m, n, k \rightarrow \infty$

\section{Definition}

The approximate point spectrum $\sigma_{a p}(T, X)=\{\alpha \in C$ : there exists (2.1), (2.2) sequence for $T-\alpha I\}$.

\section{Definition}

The defect spectrum $\sigma_{d}(T, X)=\{\alpha \in C: T-\alpha I$ is not subjective\}.

\section{Definition}

The compression spectrum $\sigma_{d} \quad(T, X)=$ $\{\alpha \in C: \overline{R(T-\alpha I)} \neq X\}$

Goldberg's Classification of Spectrum (Paul and Tripathy, 2016; Goldberg, 1985)

If $X$ is Banach metric space and $T \in B(X)$; then there are three possibilities for $R(T)$ :

- $R(T)=X$

- $R(T) \neq \overline{R(T)}=X$

- (III) $\overline{R(T)} \neq X$

- $T^{-1}$ exists and is continuous

- $T^{-1}$ exists but is discontinuous

- $T^{-1}$ does not exist

\section{Definition (Musielak, 1983)}

Let $n \in \mathbb{N}$ and $X$ be a real vector space of dimension $m$, where $n \leq m$. A real valued function $d_{p}\left(x_{1}, \ldots, x_{n}\right)=$ $\left\|\left(d_{1}\left(x_{1}, 0\right), \ldots, d_{n}\left(x_{n}, 0\right)\right)\right\|_{p}$ on $X$ satisfying the following four conditions:

- $\quad\left\|\left(d_{1}\left(x_{1}, 0\right), \ldots, d_{n}\left(x_{n}, 0\right)\right)\right\|_{p}=0$ if and only if $d_{1}\left(x_{1}\right.$, $0), \ldots, d_{n}\left(x_{n}, 0\right)$ are linearly dependent

- $\left\|\left(d_{1}\left(x_{1}, 0\right), \ldots, \quad d_{n}\left(x_{n}, 0\right)\right)\right\|_{p}$ is invariant under permutation

- $\quad\left\|\left(d_{1}\left(x_{1}, 0\right), \ldots, \alpha d_{n}\left(x_{n}, 0\right)\right)\right\|_{p}=|\alpha| \|\left(d_{1}\left(x_{1}, 0\right), \ldots, d_{n}\left(x_{n}\right.\right.$, $0)) \|_{\mathrm{p}}, \alpha \in R$

- $\quad d_{p}\left(\left(x_{1}, y_{1}\right),\left(x_{2}, y_{2}\right) \cdots\left(x_{n}, y_{n}\right)\right)=\left(d_{X}\left(x_{1}, x_{2}, \cdots x_{n}\right)^{p}+\right.$ $\left.d_{Y}\left(y_{1}, y_{2}, \cdots y_{n}\right)^{p}\right)^{1 / p}$ for $1 \leq p<\infty$

- $d\left(\left(x_{1}, y_{1}\right),\left(x_{2}, y_{2}\right), \cdots\left(x_{n}, y_{n}\right)\right):=\sup \left\{d_{X}\left(x_{1}, x_{2}, \cdots x_{n}\right)\right.$, $\left.d_{Y}\left(y_{1}, y_{2}, \cdots y_{n}\right)\right\}$, for $x_{1}, x_{2}, \cdots x_{n} \in X, y_{1}, y_{2}, \cdots y_{n} \in Y$ is called the $p$ product metric

\section{Definition}

Let $A=\left(a_{k, \ell}^{m n}\right)$ denote a four dimensional summability method that maps the complex triple 
sequences $x$ into the triple sequence $A x$ where the $k$, $\ell$-th term of $\mathrm{Ax}$ is as follows:

$$
(A x)_{k \ell}=\sum_{m=1}^{\infty} \sum_{n=1}^{\infty} \sum_{k=1}^{\infty} a_{k \ell}^{m n} x_{m n k}
$$

such transformation is said to be non-negative if $a_{k \ell}^{m n}$ is non-negative.

Let $E$ and $F$ be two sequence spaces and $A=\left(a_{k, \ell}^{m n}\right)$ be an four dimensional infinite matrix of real or complex numbers $a_{k, \ell}^{m n}$, where $m, n, k \in \mathbb{N}$. Then $A$ : $E \rightarrow F$, if for every sequence $x=\left(x_{m n k}\right)_{k \ell} \in E$ the sequence $A x=\left\{(A x)_{k \ell}\right\}$ is in $F$ where $(A x)_{k \ell}=\sum_{m=1}^{\infty} \sum_{n=1}^{\infty} \sum_{k=1}^{\infty} a_{k \ell}^{m n} x_{m n k}$, provided the right hand side converges for every $k, \ell \in \mathbb{N}$ and $x \in E$.

Consider the operator $D(p, q, r, s, t, u)$, where:

$$
D(p, q, r, s)=\left(\begin{array}{cccccccccc}
p & 0 & 0 & 0 & 0 & 0 & 0 & 0 & . & . \\
q & p & 0 & 0 & 0 & 0 & 0 & 0 & . & . \\
r & q & p & 0 & 0 & 0 & 0 & 0 & . & . \\
s & r & q & p & 0 & 0 & 0 & 0 & . & . \\
t & s & r & q & p & 0 & 0 & 0 & . & . \\
u & t & s & r & q & p & 0 & 0 & . & . \\
0 & u & t & s & r & q & p & 0 & . & . \\
. & . & . & . & . & . & . & . & . & . \\
. & . & . & . & . & . & . & . & . & . \\
. & . & . & . & . & . & . & \ddots & &
\end{array}\right)
$$

\section{Remark}

In particular if we consider $p=1, q=1, r=1, s=1, t$ $=1, u=1$ then $D(p, q, r, s, t)=\Delta_{3}$.

\section{Definition}

Let $\mathrm{f}$ be an sequence of Musielak-Orlicz functions and a sequence of spectrum operator $h$ is defined as following:

$$
\begin{aligned}
& {\left[\begin{array}{l}
\chi_{f}^{3}(\sigma(D(p, q, r, s, t))), \\
\left\|\left(d\left(x_{1}, 0\right), d\left(x_{2}, 0\right), \cdots, d\left(x_{n-1}, 0\right)\right)\right\|_{p}
\end{array}\right]=\lim _{m, n, k \rightarrow \infty}} \\
& {\left[f\left(\begin{array}{l}
\sigma(D(p, q, r, s, t))\left((m+n+k) !\left|x_{m n k}\right|\right)^{(1 / m)+n k} \\
,\left\|\left(d\left(x_{1}, 0\right), d\left(x_{2}, 0\right), \cdots, d\left(x_{n-1}, 0\right)\right)\right\|_{p}
\end{array}\right)\right]=0} \\
& {\left[\Lambda_{f}^{3}(\sigma(D(p, q, r, s, t))),\left\|\left(d\left(x_{1}, 0\right), d\left(x_{2}, 0\right), \cdots, d\left(x_{n-1}, 0\right)\right)\right\|_{p}\right]} \\
& =\sup _{m n k}\left\{\left[f\left(\begin{array}{l}
\sigma(D(p, q, r, s, t))\left|x_{m n k}\right|^{(1 / m)+n k}, \\
\left\|\left(d\left(x_{1}, 0\right), d\left(x_{2}, 0\right), \cdots, d\left(x_{n-1}, 0\right)\right)\right\|_{p}
\end{array}\right)\right]<\infty\right\}
\end{aligned}
$$

\section{Main Results}

\section{Theorem}

If $\alpha, \beta, \mu, \eta, \quad=p, q, r, s, t$ then $\alpha, \beta, \gamma, \mu$, $\eta \in \operatorname{III}_{1}\left[\chi_{f}^{3}(\sigma(D(p, q, r, s, t, u))), \|\left(d\left(x_{1}, 0\right), d\left(x_{2}\right.\right.\right.$, $\left.\left.0), \cdots, d\left(x_{n-1}, 0\right)\right) \|_{p}\right)$.

\section{Proof}

If $\alpha=p, \beta=q, \gamma=r, \mu=s, \eta=t$ then the operator $D(p, q, r, s, t, u)-\alpha I-\beta I-\gamma I-\mu I-\eta I=D(0,0,0,0,0, u)$. Since $R(D(0,0,0,0,0, u)) \neq\left[\chi_{f}^{3}(\sigma(D(p, q, r, s, t, u)))\right.$, $\left.k\left(d\left(x_{1}, 0\right), d\left(x_{2}, 0\right), \cdots, d\left(x_{n-1}, 0\right)\right) \|_{p}\right]$. It is not invertible and hence $\left[\chi_{f}^{3}(\sigma(D(p, q, r, s, t, u))), k\left(d\left(x_{1}, 0\right), d\left(x_{2}\right.\right.\right.$, $\left.\left.0), \cdots, d\left(x_{n-1}, 0\right)\right) \|_{p}\right] \in I I I_{1}$.

Therefore we have $\|\left[\chi_{f}^{3}(\sigma(D(p, q, r, s, t, u))), \|\left(d\left(x_{1}\right.\right.\right.$, $\left.\left.0), d\left(x_{2}, 0\right), \cdots, d\left(x_{n-1}, 0\right)\right) \|_{p}\right] \|=\frac{u}{3} d(x, 0)$. It is bounded below and it has a bounded inverse. Hence $\alpha, \beta, \gamma, \mu$, $\eta \in I I I_{1}\left[\chi_{f}^{3}(\sigma(D(p, q, r, s, t, u))), \|\left(d\left(x_{1}, 0\right), d\left(x_{2}, 0\right), \cdots\right.\right.$, $\left.\left.d\left(x_{n-1}, 0\right)\right) \|_{p}\right]$. This completes the proof.

\section{Lemma:}

$$
\begin{aligned}
& {\left[\chi_{f}^{3}(\sigma(D(p, q, r, s, t, u))),\left\|\left(d\left(x_{1}, 0\right), d\left(x_{2}, 0\right), \cdots, d\left(x_{n-1}, 0\right)\right)\right\|_{p}\right)} \\
& =\{\alpha, \beta, \gamma, \mu, \eta \in \mathbb{C}:|\alpha-p|,|\beta-q|,|\gamma-r \| \mu-s|,|\eta-t| \leq|u|\}
\end{aligned}
$$

\section{Theorem:}

$$
\begin{aligned}
& {\left[\begin{array}{l}
\chi_{f}^{3}\left(\sigma_{a p}(D(p, q, r, s, t, u))\right), \\
\left\|\left(d\left(x_{1}, 0\right), d\left(x_{2}, 0\right), \cdots, d\left(x_{n-1}, 0\right)\right)\right\|_{p}
\end{array}\right]} \\
& =\left\{\begin{array}{l}
\alpha, \beta, \gamma, \mu, \eta \in \mathbb{C}:|\alpha-p|,|\beta-q|, \\
|\gamma-r|,|\mu-s|,|\eta-t| \leq|u|
\end{array}\right\} \backslash\{p\},\{q\},\{r\},\{s\},\{t\}
\end{aligned}
$$

\section{Proof}

\section{We have:}

$$
\begin{aligned}
& {\left[\chi_{f}^{3}\left(\sigma_{a p}(D(p, q, r, s, t, u))\right),\left\|\left(d\left(x_{1}, 0\right), d\left(x_{2}, 0\right), \cdots, d\left(x_{n-1}, 0\right)\right)\right\|_{p}\right)} \\
& =\left[\chi_{f}^{3}(\sigma(D(p, q, r, s, t, u))),\left\|\left(d\left(x_{1}, 0\right), d\left(x_{2}, 0\right), \cdots, d\left(x_{n-1}, 0\right)\right)\right\|_{p}\right) \backslash \\
& \operatorname{III}_{1}\left[\chi_{f}^{3}(\sigma(D(p, q, r, s, t, u))),\left\|\left(d\left(x_{1}, 0\right), d\left(x_{2}, 0\right), \cdots, d\left(x_{n-1}, 0\right)\right)\right\|_{p}\right), \\
& {\left[\chi_{f}^{3}\left(\sigma_{a p}(D(p, q, r, s, t, u))\right),\left\|\left(d\left(x_{1}, 0\right), d\left(x_{2}, 0\right), \cdots, d\left(x_{n-1}, 0\right)\right)\right\|_{p}\right)} \\
& =\{\alpha, \beta, \gamma, \mu, \eta \in \mathbb{C}:|\alpha-p|,|\beta-q|,|\gamma-r|,|\mu-s|,|\eta-t| \leq|u|\} \backslash \\
& \{p\},\{q\},\{r\},\{s\},\{t\}
\end{aligned}
$$


is obtained by Lemma (3.2) and Theorem (3.1). This completes the proof.

Lemma:

$$
\left[\begin{array}{l}
\chi_{f}^{3}\left(\sigma_{p}(D(p, q, r, s, t, u))\right), \\
\left.\|\left(d\left(x_{1}, 0\right), d\left(x_{2}, 0\right), \cdots, d\left(x_{n-1}, 0\right)\right)\right) \|_{p}
\end{array}\right)=\phi
$$

\section{Theorem:}

$\left[\chi_{f}^{3}\left(\sigma_{d}(D(p, q, r, s, t, u))\right),\left\|\left(d\left(x_{1}, 0\right), d\left(x_{2}, 0\right), \cdots, d\left(x_{n-1}, 0\right)\right)\right\|_{p}\right)$ $=\{\alpha, \beta, \gamma, \mu, \eta \in \mathbb{C}:|\alpha-p|,|\beta-q|,|\gamma-r|,|\mu-s|,|\eta-t| \leq|u|\} \backslash$ $\{p\},\{q\},\{r\},\{s\},\{t\}$

\section{Proof}

We have:

$\left[\chi_{f}^{3}\left(\sigma_{d}(D(p, q, r, s, t, u))\right),\left\|\left(d\left(x_{1}, 0\right), d\left(x_{2}, 0\right), \cdots, d\left(x_{n-1}, 0\right)\right)\right\|_{p}\right)$ $=\left[\chi_{f}^{3}(\sigma(D(p, q, r, s, t, u))),\left\|\left(d\left(x_{1}, 0\right), d\left(x_{2}, 0\right), \cdots, d\left(x_{n-1}, 0\right)\right)\right\|_{p}\right) \backslash$ $I_{3}\left[\chi_{f}^{3}(\sigma(D(p, q, r, s, t, u))),\left\|\left(d\left(x_{1}, 0\right), d\left(x_{2}, 0\right), \cdots, d\left(x_{n-1}, 0\right)\right)\right\|_{p}\right)$

Now:

$I_{3}\left[\chi_{f}^{3}(\sigma(D(p, q, r, s, t, u))),\left\|\left(d\left(x_{1}, 0\right), d\left(x_{2}, 0\right), \cdots, d\left(x_{n-1}, 0\right)\right)\right\|_{p}\right)$ $=I I_{3}\left[\chi_{f}^{3}(\sigma(D(p, q, r, s, t, u))),\left\|\left(d\left(x_{1}, 0\right), d\left(x_{2}, 0\right), \cdots, d\left(x_{n-1}, 0\right)\right)\right\|_{p}\right)$ $=I I I I_{3}\left[\chi_{f}^{3}(\sigma(D(p, q, r, s, t, u))),\left\|\left(d\left(x_{1}, 0\right), d\left(x_{2}, 0\right), \cdots, d\left(x_{n-1}, 0\right)\right)\right\|_{p}\right)$ $=\left[\chi_{f}^{3}\left(\sigma_{p}(D(p, q, r, s, t, u))\right),\left\|\left(d\left(x_{1}, 0\right), d\left(x_{2}, 0\right), \cdots, d\left(x_{n-1}, 0\right)\right)\right\|_{p}\right)=\phi$

by Lemma (3.4). Hence:

$$
\begin{aligned}
& {\left[\chi_{f}^{3}\left(\sigma_{d}(D(p, q, r, s, t, u))\right),\left\|\left(d\left(x_{1}, 0\right), d\left(x_{2}, 0\right), \cdots, d\left(x_{n-1}, 0\right)\right)\right\|_{p}\right)} \\
& =\left[\chi_{f}^{3}(\sigma(D(p, q, r, s, t, u))),\left\|\left(d\left(x_{1}, 0\right), d\left(x_{2}, 0\right), \cdots, d\left(x_{n-1}, 0\right)\right)\right\|_{p}\right)
\end{aligned}
$$

\section{Lemma:}

$\left[\chi_{f}^{3}\left(\sigma_{r}(D(p, q, r, s, t, u))\right),\left\|\left(d\left(x_{1}, 0\right), d\left(x_{2}, 0\right), \cdots, d\left(x_{n-1}, 0\right)\right)\right\|_{p}\right)$ $=\{\alpha, \beta, \gamma, \mu, \eta \in \mathbb{C}:|\alpha-p|,|\beta-q|,|\gamma-r|,|\mu-s|,|\eta-t| \leq|u|\}$

\section{Theorem:}

$\left[\chi_{f}^{3}\left(\sigma_{c o}(D(p, q, r, s, t, u))\right),\left\|\left(d\left(x_{1}, 0\right), d\left(x_{2}, 0\right), \cdots, d\left(x_{n-1}, 0\right)\right)\right\|_{p}\right)$ $=\{\alpha, \beta, \gamma, \mu, \eta \in \mathbb{C}:|\alpha-p|,|\beta-q|,|\gamma-r|,|\mu-s|,|\eta-t| \leq|s|\} \backslash$ $\{p\},\{q\},\{r\},\{s\},\{t\}$

\section{Proof:}

$\left[\chi_{f}^{3}\left(\sigma_{c_{0}}(D(p, q, r, s, t, u))\right),\left\|\left(d\left(x_{1}, 0\right), d\left(x_{2}, 0\right), \cdots, d\left(x_{n-1}, 0\right)\right)\right\|_{p}\right)$ $=I I I_{1}\left[\chi_{f}^{3}(\sigma(D(p, q, r, s, t, u))),\left\|\left(d\left(x_{1}, 0\right), d\left(x_{2}, 0\right), \cdots, d\left(x_{n-1}, 0\right)\right)\right\|_{p}\right) \mathrm{U}$ III $2\left[\chi_{f}^{3}(\sigma(D(p, q, r, s, t, u))),\left\|\left(d\left(x_{1}, 0\right), d\left(x_{2}, 0\right), \cdots, d\left(x_{n-1}, 0\right)\right)\right\|_{p}\right) \cup$ $\operatorname{III}_{3}\left[\chi_{f}^{3}(\sigma(D(p, q, r, s, t, u))),\left\|\left(d\left(x_{1}, 0\right), d\left(x_{2}, 0\right), \cdots, d\left(x_{n-1}, 0\right)\right)\right\|_{p}\right)$

Now:

$I I I_{1}\left[\chi_{f}^{3}(\sigma(D(p, q, r, s, t, u))),,\left(\left(d\left(x_{1}, 0\right), d\left(x_{2}, 0\right), \cdots, d\left(x_{n-1}, 0\right)\right) \|_{p}\right) \bigcup\right.$ $\operatorname{III}_{2}\left[\chi_{f}^{3}(\sigma(D(p, q, r, s, t, u))), \cdot\left(d\left(x_{1}, 0\right), d\left(x_{2}, 0\right), \cdots, d\left(x_{n-1}, 0\right)\right) \|_{p}\right)$ $=\left[\chi_{f}^{3}\left(\sigma_{r}(D(p, q, r, s, t, u))\right),\left\|\left(d\left(x_{1}, 0\right), d\left(x_{2}, 0\right), \cdots, d\left(x_{n-1}, 0\right)\right)\right\|_{p}\right)$ $=\{\alpha, \beta, \gamma, \mu, \eta \in \mathbb{C}:|\alpha-p|,|\beta-q|,|\gamma-r|,|\mu-s|,|\eta-t| \unlhd u \mid\}$

is obtained by Lemma (3.6). Again:

$$
\begin{aligned}
& \operatorname{III}_{3}\left[\chi_{f}^{3}(\sigma(D(p, q, r, s, t, t u))),\left\|\left(d\left(x_{1}, 0\right), d\left(x_{2}, 0\right), \cdots, d\left(x_{n-1}, 0\right)\right)\right\|_{p}\right) \\
& =\left[\chi_{f}^{3}\left(\sigma_{p}(D(p, q, r, s, t, u))\right), \cdot\left(\left(d\left(x_{1}, 0\right), d\left(x_{2}, 0\right), \cdots, d\left(x_{n-1}, 0\right)\right) \|_{p}\right)=\phi\right.
\end{aligned}
$$

is obtained by Lemma (3.4). Hence:

$\left[\chi_{f}^{3}\left(\sigma_{c_{0}}(D(p, q, r, s, t, u))\right),\left\|\left(d\left(x_{1}, 0\right), d\left(x_{2}, 0\right), \cdots, d\left(x_{n-1}, 0\right)\right)\right\|_{p}\right)$
$=\{\alpha, \beta, \gamma, \mu, \eta \in \mathbb{C}:|\alpha-p|,|\beta-q|,|\gamma-r|,|\mu-s|,|\eta-t| \leq|u|\} \backslash$
$\{p\},\{q\},\{r\},\{s\},\{t\}$

This completes the proof.

\section{Lemma}

The adjoint operator $T^{*}$ of $T$ is onto if and only if $T$ has a bounded inverse.

\section{Theorem}

If $\alpha, \beta, \gamma, \mu, \eta=p, q, r, s, t, u$ then: 


$$
\begin{aligned}
& \alpha, \beta, \gamma, \mu, \eta \in \\
& \operatorname{III}_{1}\left[\chi_{f}^{3}(\sigma(D(p, q, r, s, t, u))),\left\|\left(d\left(x_{1}, 0\right), d\left(x_{2}, 0\right), \cdots, d\left(x_{n-1}, 0\right)\right)\right\|_{p}\right)
\end{aligned}
$$

\section{Proof}

By Lemma (3.6):

$$
\begin{aligned}
& \alpha, \beta, \gamma, \mu, \eta \in \\
& \operatorname{III}_{1}\left[\chi_{f}^{3}(\sigma(D(p, q, r, s, t, u))),\left\|\left(d\left(x_{1}, 0\right), d\left(x_{2}, 0\right), \cdots, d\left(x_{n-1}, 0\right)\right)\right\|_{p}\right)
\end{aligned}
$$

whenever $\alpha=p, \beta=q, \gamma=r, \mu=s, \eta=t$. By Lemma (3.8), $\alpha=p, \beta=q, \gamma=r, \mu=s, \eta=t$ is not in $\left[\chi_{f}^{3}(\sigma(D(p, q, r, s, t, u))),\left\|\left(d\left(x_{1}, 0\right), d\left(x_{2}, 0\right), \cdots, d\left(x_{n-1}, 0\right)\right)\right\|_{p}\right)$ and hence:

$$
\left(\left[\begin{array}{l}
{\left[\chi_{f}^{3}(\sigma(D(p, q, r, s, t, u))),\left\|\left(d\left(x_{1}, 0\right), d\left(x_{2}, 0\right), \cdots, d\left(x_{n-1}, 0\right)\right)\right\|_{p}\right)} \\
-\alpha I, \beta I, \gamma I, \mu I, \eta I
\end{array}\right)^{-1}\right.
$$

exists. We have to prove that:

$$
\left.\left(\begin{array}{l}
{\left[\chi_{f}^{3}(\sigma(D(p, q, r, s, t, u))),\left\|\left(d\left(x_{1}, 0\right), d\left(x_{2}, 0\right), \cdots, d\left(x_{n-1}, 0\right)\right)\right\|_{p}\right)} \\
-\alpha I, \beta I, \gamma I, \mu I, \eta I
\end{array}\right)\right)^{-1}
$$

must be continuous, hence it is show that:

$$
\begin{aligned}
& {\left[\chi_{f}^{3}(\sigma(D(0,0,0,0,0, u))),\left\|\left(d\left(x_{1}, 0\right), d\left(x_{2}, 0\right), \cdots, d\left(x_{n-1}, 0\right)\right)\right\|_{p}\right)^{*}} \\
& -\alpha I, \beta I, \gamma I, \mu I, \eta I \\
& =\left[\chi_{f}^{3}(\sigma(D(0,0,0,0,0, u))),\left\|\left(d\left(x_{1}, 0\right), d\left(x_{2}, 0\right), \cdots, d\left(x_{n-1}, 0\right)\right)\right\|_{p}\right)^{*}
\end{aligned}
$$

is onto by Lemma (3.8). Given:

$$
y=\left(y_{\text {mnk }}\right) \in\left[\begin{array}{l}
\Lambda_{f}^{3}(\sigma(D(0,0,0,0,0, u))), \\
\left.\|\left(d\left(x_{1}, 0\right), d\left(x_{2}, 0\right), \cdots, d\left(x_{n-1}, 0\right)\right)\right) \|_{p}
\end{array}\right)
$$

we can find:

$$
x=\left(x_{m n k}\right) \in\left[\begin{array}{l}
\Lambda_{f}^{3}(\sigma(D(0,0,0,0,0, u))), \\
\left\|\left(d\left(x_{1}, 0\right), d\left(x_{2}, 0\right), \cdots, d\left(x_{n-1}, 0\right)\right)\right\|_{p}
\end{array}\right)
$$

such that:

$$
\begin{aligned}
& {\left[\chi_{f}^{3}(\sigma(D(0,0,0,0,0, u))),\left\|\left(d\left(x_{1}, 0\right), d\left(x_{2}, 0\right), \cdots, d\left(x_{n-1}, 0\right)\right)\right\|_{p}\right)} \\
& =\left[\Lambda_{f}^{3}(\sigma(D(0,0,0,0,0, u))),\left\|\left(d\left(x_{1}, 0\right), d\left(x_{2}, 0\right), \cdots, d\left(x_{n-1}, 0\right)\right)\right\|_{p}\right)
\end{aligned}
$$

Therefore we have:

$$
x_{m n k}=\frac{1}{u} y_{m-1, n-2, k-3}
$$

which shows that:

$$
\left[\chi_{f}^{3}(\sigma(D(0,0,0,0,0, u))),\left\|\left(d\left(x_{1}, 0\right), d\left(x_{2}, 0\right), \cdots, d\left(x_{n-1}, 0\right)\right)\right\|_{p}\right)^{*}
$$

is onto. This completes the proof.

\section{Conclusion}

Author's are introduced and examined various spectrum of the operator $D(p, q, r, s, t, u)$ on the sequence space $\chi^{3}$ defined by Musielak-Orlicz function.

\section{Acknowledgment}

The authors are extremely grateful to the anonymous learned referee(s) for their keen reading, valuable suggestion and constructive comments for the improvement of the manuscript. The authors are thankful to the editor(s) and reviewers of Journal of Mathematics and Statistics.

\section{Author's Contributions}

First author study using the concept of generalized difference operator are introduced and second author examined various spectrum of the operator $D(p, q, r, s, t$, $u$ ) on the sequence space $\chi^{3}$ defined by Musielak-Orlicz function. Moreover, we have established some relations concerning with this space.

\section{Competing Interests}

The authors declare that there is not any conflict of interests regarding the publication of this manuscript.

\section{References}

Apostol, T., 1978. Mathematical Analysis. 1st Edn., Addison-Wesley, London.

Deepmala, N.S. and V.N. Mishra, 2016. Double almost $\left(\lambda_{m} \mu_{n}\right)$ in $\chi^{2}$-Riesz space. Southeast Asian Bull. Math.

Deepmala, R., L.N. Mishra and N. Subramanian, 2016. Characterization of some Lacunary $\chi_{A u v^{-}}$ convergence of order $\alpha$ with $p$-metric defined by $m n$ sequence of moduli Musielak, Applied Math. Inf. Sci. Lett., 4: 119-126. DOI: 10.18576/amis1/040304 
Esi, A. and E. Savas, 2015. On lacunary statistically convergent triple sequences in probabilistic normed space. Applied Math. Infrom. Sci., 9: 2529-2534. DOI: $10.12785 / \mathrm{amis} / 090537$

Esi, A. and M. Necdet Catalbas, 2014. Almost convergence of triple sequences. Global J. Math. Anal., 2: 6-10. DOI: 10.14419/gjma.v2i1.1709

Esi, A., 2014. On some triple almost lacunary sequence spaces defined by Orlicz functions. Res. Rev.: Discrete Math. Struct., 1: 16-25.

Goldberg, S., 1985. Unbounded Linear Operators: Theory and Applications. 1st Edn., Dover Publications Inc., New York, ISBN-10: 0486648303, pp: 199.

Hardy, G.H., 1917. On the convergence of certain multiple series. Proc. Camb. Phil. Soc., 19: 86-95.

Kamthan, P.K. and M. Gupta, 1981. Sequence Spaces and Series. 1st Edn., M. Dekker, New York, ISBN-10: 0824712242, pp: 368.

Kizmaz, H., 1981. On certain sequence spaces. Canad. Math. Bull., 24: 169-176.

DOI: 10.4153/CMB-1981-027-5
Lindenstrauss, J. and L. Tzafriri, 1971. On Orlicz sequence spaces. Israel J. Math., 10: 379-390. DOI: $10.1007 / \mathrm{BF} 02771656$

Musielak, J., 1983. Orlicz Spaces and Modular Spaces. 1 st Edn., Springer, New York, ISBN-10: 3540127062, pp: 222.

Paul, A. and B.C. Tripathy, 2016. Subdivisions of the spectra for the operator $\mathrm{D}(\mathrm{r}, 0,0, \mathrm{~s})$ over certain sequence spaces. Bol. Soc. Paran. Mat., 3: 75-84. DOI: $10.5269 /$ bspm.v34i1.22759

Sahiner, A., M. Gurdal and F.K. Duden, 2007. Triple sequences and their statistical convergence. Selcuk J. Applied Math., 8: 49-55.

Prakash, T.V.G.S., M. Chandramouleeswaran and N. Subramanian, 2016. Lacunary Triple sequence $\Gamma_{3}$ of Fibonacci numbers over probabilistic p-metric spaces. Int. Organiz. Scientific Res., 12: 10-16. www.iosrjournals.org

Subramanian, N. and A. Esi, 2015. Some new seminormed triple sequence spaces defined by a sequence of moduli. J. Anal. Number Theory, 3: 79-88. DOI: $10.18576 /$ jant/030207 